\title{
Models for the study of electronic excitation and transfer processes.
}

\author{
Paula Homem-de-Mello ${ }^{1}$ (PQ) \\ paula.mello@ufabc.edu.br \\ ${ }^{1}$ Centro de Ciências Naturais e Humanas, Universidade Federal do ABC, Santo André, SP, Brazil. \\ Palavras-chave: electronic transfer and excitation, DFT, MD, sensors, photodynamic therapy.
}

When experimentalists propose us a problem, looking for a microscopic view of the processes that are occurring in their experimental arrangement, these problems often involve complex systems with a high number of variables. How to deal with that? Which approach is the more adequate to be employed? Of course, there is not a sole answer. Here, it will be presented how our group is dealing with different problems, particularly aiming to contribute to the understanding of excitation and transfer of electrons.

Electronic excitations are the basis of the use of dyes in different systems: from dyesensitized solar cells (DSSC) to applications in the treatment of diseases, such as photodynamic therapy (PDT). Proposing dyes with higher efficiency for these applications is a joint venture between theorists and experimentalists. For both applications, basically the dye absorbs light radiation and transforms that energy into electrical current, in the case of DSSCs, or in free radicals, in the case of PDT. Thus, understanding the states involved in electronic excitation is the first step in planning for new molecules. Understanding what happens to the electron next and how the medium affects the decay and electron transfer processes are the next steps.

Another system we are very interested in is the electrochemical drug sensors. These are quite complex systems too, because involves a substrate, composed by nanostructures and ionic liquids, and adsorption and desorption steps may be studied.

Many methodologies can be employed to study those systems, and we have to make an assessment of their cost-effectiveness. Particularly, the choice and construction of the model is a very important process, which will define the quality of information we are obtaining, and the validity of the correlation between computational and experimental data. In the seminar, some of the tests that we have done as well as our findings will be presented.

Acknowledgements: FAPESP and CNPq.

References:

1. De Carvalho, F. et al. Density Functional Theory Applied to Excited State Intramolecular Proton Transfer in Imidazole-, Oxazole-, and Thiazole-Based Systems. Molecules, 23, 1231, 2018.

2. Bettanin, F. et al. Quantum-chemistry descriptors for photosensitizers based on macrocycles. Chem Bio \& Drug Design, 89, 207-220, 2017.

3. Sousa, C. P. et al. Chlorhexidine digluconate on chitosan-magnetic iron oxide nanoparticles modified electrode: Electroanalysis and mechanistic insights by computational simulations. Sensors and Actuators. B, Chemical, 240, 417-425, 2017.

4. Bettanin, F. et al. A quantum chemical investigation of the solvatochromism of a phthalocyanine within a lipid bilayer: Comparison between continuum and atomistic models. J. Photochem Photobio A-Chemistry, 344, 42-48, 2017. 